\title{
Uncertainty Quantification in $(\alpha, n)$ Neutron Source Calculations for an Oxide Matrix
}

\author{
M. T. Pigni, ${ }^{1, *}$ S. Croft,${ }^{1, \dagger}$ and I. C. Gauld ${ }^{1, \ddagger}$ \\ ${ }^{1}$ Oak Ridge National Laboratory, Oak Ridge, TN 37831-6170, USA ${ }^{\S}$
}

(Dated: March 22, 2016)

\begin{abstract}
We present a methodology to propagate nuclear data covariance information in neutron source calculations from $(\alpha, \mathrm{n})$ reactions. The approach is applied to estimate the uncertainty in the neutron generation rates for uranium oxide fuel types due to uncertainties on 1$){ }^{17,18} \mathrm{O}(\alpha, \mathrm{n})$ reaction crosssections and 2) uranium and oxygen stopping power cross sections.

The procedure to generate reaction cross section covariance information is based on the Bayesian fitting method implemented in the $R$-matrix SAMMY code. The evaluation methodology uses the Reich-Moore approximation to fit the ${ }^{17,18} \mathrm{O}(\alpha, \mathrm{n})$ reaction cross-sections in order to derive a set of resonance parameters and a related covariance matrix that is then used to calculate the energydependent cross section covariance matrix. The stopping power cross sections and related covariance information for uranium and oxygen were obtained by the fit of stopping power data in the $\alpha$-energy range of $1 \mathrm{keV}$ up to $12 \mathrm{MeV}$.

Cross section perturbation factors based on the covariance information relative to the evaluated ${ }^{17,18} \mathrm{O}(\alpha, \mathrm{n})$ reaction cross sections, as well as uranium and oxygen stopping power cross sections, were used to generate a varied set of nuclear data libraries used in SOURCES4C and ORIGEN for inventory and source term calculations. The set of randomly perturbed output $(\alpha, n)$ source responses, provide the mean values and standard deviations of the calculated responses reflecting the uncertainties in nuclear data used in the calculations. The results and related uncertainties are compared with experiment thick target $(\alpha, \mathrm{n})$ yields for uranium oxide.
\end{abstract}

Keywords: nuclear data, $R$-matrix theory, covariance data, neutron source

\section{INTRODUCTION}

Nuclear data describing $\alpha$-particle interactions on light nuclei are essential for calculating neutron emission via $(\alpha, n)$ processes. Limited to oxide fuel, this work focuses on ${ }^{17,18} \mathrm{O}(\alpha, \mathrm{n})$ cross sections and describes a methodology to estimate uncertainty in $(\alpha, n)$ neutron source calculations due to uncertainty in nuclear data. The impact of the uncertainty related to both ${ }^{17,18} \mathrm{O}(\alpha, \mathrm{n})$ cross sections and stopping power cross sections of oxygen and uranium propagated to neutron source generation is in-

\footnotetext{
* Corresponding author: pignimt@ornl.gov

† crofts@ornl.gov

$\ddagger$ gauldi@ornl.gov

$\S$ Notice: This manuscript has been authored by UT-Battelle, LLC under Contract No. DE-AC05-00OR22725 with the U.S. Department of Energy. The United States Government retains and the publisher, by accepting the article for publication, acknowledges that the United States Government retains a non-exclusive, paidup, irrevocable, world-wide license to publish or reproduce the published form of this manuscript, or allow others to do so, for United States Government purposes. The Department of Energy will provide public access to these results of federally sponsored research in accordance with the DOE Public Access Plan (http://energy.gov/downloads/doe-public-access-plan).
}

vestigated using the ORIGEN code [1] in a typical uranium oxide fuel type. An inventory code widely used to predict the properties of irradiated fuel, ORIGEN adopts the methods used in the SOURCES4C code [2][3], which is widely used in nuclear safeguards, spent nuclear fuel characterization, and neutron source descriptions due to spontaneous fission and $(\alpha, \mathrm{n})$ reactions.

Neutron emission by $(\alpha, n)$ reactions can represent a significant neutron source in neutron-based verification measurements of unirradiated uranium and plutonium nuclear materials in a non-metal matrix. In irradiated nuclear fuels that achieve a moderate to high burnup, the neutron source is typically dominated by ${ }^{242,244} \mathrm{Cm}$ spontaneous fission. However, in low-burnup fuels such as those for weapons material production, the $(\alpha, n)$ neutron processes can represent a large component of the total neutron source and are therefore important to neutron measurement systems.

Currently, in the SOURCES4C and ORIGEN libraries, there is no information on covariance data or even data uncertainties. Therefore, no uncertainty is provided, for instance, in the estimated neutron source intensities or in the emission spectra. Among cross section data in the SOURCES4C and ORIGEN libraries, those concerning $\alpha$-particle interactions with oxygen are based on Bair's thin- and thick-target measurements [4][5][6] for 
the lower-energy and on Hansen's data [7] for the highenergy range. Babut [8] previously described a methodology used for analyzing Bair's experimental cross sections in the framework of the Reich-Moore formalism with the Bayesian fitting code SAMMY. However, the ultimate objective of that work was primarily to quantify the impact of the resonance structure on the neutron energy spectrum produced by $(\alpha, n)$ reactions, and no uncertainty quantification on the neutron source term was performed.

In this work, the procedure to generate nuclear data covariance information from experimental data is based on the $R$-matrix code SAMMY [9]. The evaluation methodology uses the Reich-Moore approximation to compute the ${ }^{17,18} \mathrm{O}(\alpha, \mathrm{n})$ reaction cross sections and the Bayesian fitting method to generate a set of resonance parameters with a related covariance matrix in the incident $\alpha$ energy range up to $\gtrsim 5 \mathrm{MeV}$, i.e., the upper range of Bair's measurements. Above $\approx 5-10 \mathrm{MeV}$, we adopted for ${ }^{17} \mathrm{O}$ the evaluated reaction cross sections found in the JENDL nuclear data library [10].

The resonance parameter covariance matrix was then used to calculate the energy-dependent cross section covariance matrix up to $5.14 \mathrm{MeV}$. For incident energy above $5.14 \mathrm{MeV}$ up $10 \mathrm{MeV}$, a constant $20 \%$ uncertainty was assigned to the JENDL pointwise ${ }^{17} \mathrm{O}(\alpha, \mathrm{n})$ cross sections and the point-to-point values were taken to be not correlated (diagonal covariance matrix). The uncertainty for ${ }^{18} \mathrm{O}(\alpha, \mathrm{n})$ based on the accuracy of ${ }^{\text {nat }} \mathrm{O}$ cross sections was estimated at $1.5 \%$ based on the review of [11].

To quantify the contribution of the stopping power cross sections of the materials used in ORIGEN calculations, we also derived a set of parameters and related covariance information by fitting the data found in the ASTAR database [12] over the $\alpha$-energy range of $1 \mathrm{keV}$ up to $12 \mathrm{MeV}$.

A set of random perturbation factors was obtained by stochastic sampling of the multivariate (normal) distributions [3] based on the covariance matrix relative to both evaluated ${ }^{17,18} \mathrm{O}(\alpha, \mathrm{n})$ cross sections and fit coefficients used to compute the oxygen and uranium stopping power cross sections. The set of perturbed cross sections was then used in ORIGEN calculations to obtain, by statistical analysis, the mean value and related uncertainty for the specific case of $(\alpha, \mathrm{n})$ source and stopping power cross sections combined.

\section{THEORY OF NEUTRON SOURCE CALCULATIONS}

ORIGEN incorporates a variety of data included in precompiled data libraries for the stopping power cross sections and $(\alpha, \mathrm{n})$ reaction cross sections (compiled from experimental data or based on nuclear physics model calculations), as well as information related to the decay processes such as branching ratios, spontaneous fission decay constants, and $\alpha$-particle emission energies and yields. These data and methods in ORIGEN are directly adopted from the SOURCES4C code.

In this section we define the theory leading to $(\alpha, \mathrm{n})$ reactions and the related neutron production function. This is relevant to clarify the relationship between the $(\alpha, \mathrm{n})$ neutron source and the nuclear data, including $(\alpha, \mathrm{n})$ reaction cross sections and stopping power cross sections.

In particular, we briefly describe the model to calculate the average number of neutrons emitted from $(\alpha, n)$ reactions by the definition of thick-target neutron production function,

$$
p_{i}\left(E_{\alpha}\right)=\frac{N_{i}}{N} \int_{0}^{E_{\alpha}} \sigma_{i}(E) \epsilon(E)^{-1} \mathrm{~d} E .
$$

This quantity represents the probability that an $\alpha$ particle with energy $E_{\alpha}$ will undergo an $(\alpha, n)$ reaction with the nuclide $i$ normalized by the ratio of the atom density of nuclide $N_{i}$ and the total density of the material,

$$
N=\sum_{i=1}^{k} N_{i},
$$

for a material composed by $k$ elemental constituents. The integrand of Eq. (1) is defined by $\sigma_{i}(E)$, the $(\alpha, n)$ cross section (in barns) for nuclide $i$, and $\epsilon(E)$, the stopping cross section (in $\mathrm{eV} / 10^{15} / \mathrm{cm}^{2}$ ). The latter is approximated by the Bragg-Kleeman [13] relationship

$$
\epsilon(E) \cong \frac{1}{N} \sum_{i=1}^{k} N_{i} \epsilon_{i}(E),
$$

where $\epsilon_{i}(E)$ is the stopping cross section for nuclide $i$. The integral is computed between the initial energy of the $\alpha$-particle, $E=E_{\alpha}$, and $E=0$ when the $\alpha$-particle has completely lost its energy, i.e. stopped.

A fraction of the decays of nuclide $i$ within the material is by $\alpha$ emission. This fraction,

$$
F_{i}^{\alpha}=\sum_{j=1}^{m} f_{i}^{\alpha}\left(E_{\alpha_{j}}\right),
$$

can occur with the emission of one of $m$ possible discrete $\alpha$-energies $E_{\alpha_{j}}, j=1, \ldots, m$ of intensity $f_{i j}^{\alpha}$. Thus, the fraction of nuclide $i^{\prime}$ decays resulting in $(\alpha, n)$ neutron production in a thick-target material containing $k$ nuclides with $(\alpha, n)$ cross section is

$$
R_{i^{\prime}}=\sum_{j=1}^{m} f_{i^{\prime}}\left(E_{\alpha_{j}}\right) \sum_{i=1}^{k} p_{i}\left(E_{\alpha_{j}}\right) .
$$

Equation (1) shows that $\sigma(E)$ and $\epsilon(E)$ are the only two nuclear data components contributing to the uncertainties, assuming the stoichiometry of the matrix compound is known. 


\section{EVALUATION METHODOLOGY}

The SAMMY code system [9] is a modern tool for calculating reaction cross sections mainly used for nuclear data evaluations in the neutron resolved resonance region. However, SAMMY has built-in capabilities that also allow the code to evaluate cross sections for other incident particles including, charged particles [8]. The SAMMY code incorporates selected $R$-matrix approximations coupled to the Bayesian method to fit experimental data and ultimately to generate a set of resonance parameters with a related parameter covariance matrix. For this task, we based our results on the Reich-Moore formalism, which approximates the expression for elastic and reaction cross sections better than other single- and multi-level variants of the $R$-matrix theory.

We will not describe the $R$-matrix theory; we simply refer to Fröhner's report [14] and Pigni's paper [15] for a concise but complete introduction to the principles of the resonance theory. Moreover, in this paper we will mainly focus on the application of our methodology to the evaluation of $\alpha$-particle cross sections.

\section{A. Thin-target experimental data}

To show the feasibility of the method, thin-target ${ }^{17,18} \mathrm{O}(\alpha, \mathrm{n})$ cross sections measured by Bair [4][5] were used to estimate a preliminary set of resonance parameters and a related covariance matrix. As displayed in Table I, the analysis of the ${ }^{18} \mathrm{O}$ isotope was based on two thin-target measurements of $(\alpha, \mathrm{n})$ cross section data. The first set covers the incident $\alpha$-energy range 1.06 $\leq E_{\alpha} \leq 2.50 \mathrm{MeV}$, and the second covers $2.43 \leq E_{\alpha} \leq$ $5.14 \mathrm{MeV}$. Experimental data for natural oxygen were used to evaluate ${ }^{18} \mathrm{O}(\alpha, \mathrm{n})$ cross sections above $5.14 \mathrm{MeV}$.

TABLE I. ${ }^{17,18} \mathrm{O}(\alpha, \mathrm{n})$ experimental cross section data sets used in SAMMY calculations. Experimental data set on natural oxygen to evaluate ${ }^{18} \mathrm{O}(\alpha, \mathrm{n})$ cross sections above 5.14 $\mathrm{MeV}$.

\begin{tabular}{cccccc}
\hline Nucleus & Author & $\mathrm{E}_{\alpha}(\mathrm{MeV})$ & Unc.(\%) & Norm. ${ }^{\mathrm{a}}$ & Unc. $(\%)^{\mathrm{b}}$ \\
\hline${ }^{17} \mathrm{O}$ & Bair [5] & $0.92-5.31$ & $25^{\mathrm{c}}$ & 1.35 & 7 \\
${ }^{18} \mathrm{O}$ & Bair [4] & $1.06-2.50$ & $25^{\mathrm{d}}$ & 1.35 & 7 \\
${ }^{18} \mathrm{O}$ & Bair [5] & $2.43-5.14$ & 25 & 1.35 & 7 \\
${ }^{n a t} \mathrm{O}$ & Bair [6] & $4.62-4.8^{\mathrm{e}}$ & 7 & 1.00 & 7 \\
${ }^{\text {nat } \mathrm{O}}$ & West [11] & $3.80-10.0$ & 1.5 & 1.00 & 1.5 \\
\hline
\end{tabular}

${ }^{a}$ Normalization factors recommended by Bair to correct bias in thin-target measurements.

b Uncertainty assigned to each data point after normalization as stated in [6]. No correlation between data points was assumed.

${ }^{c}$ Excluding uncertainty of graphite sphere detector efficiency.

d Assumed uncertainty since no error analysis was reported.

e Range of yield measurements.

Since, in the analyzed energy range, ${ }^{18} \mathrm{O}$ dominates neutron emission ${ }^{1}$ in ${ }^{\text {nat }} \mathrm{O}$ because of both its isotopic abundance and larger $(\alpha, \mathrm{n})$ cross section than ${ }^{17} \mathrm{O}$, the evaluation of the ${ }^{18} \mathrm{O}$ reaction cross sections $\gtrsim 5 \mathrm{MeV}$ was based on a high-quality experimental data set of natural oxygen [11].

Bair's measurement for the ${ }^{17} \mathrm{O}$ isotope extends over the energy range $0.92 \leq E_{\alpha} \leq 5.31 \mathrm{MeV}$. The reported overall uncertainty for ${ }^{17,18} \mathrm{O}$ experimental data sets [5] measured up to about $5 \mathrm{MeV}$ was reported as at least $25 \%$. The same percentage of uncertainty was assumed for the ${ }^{18} \mathrm{O}$ data set [4] measured at $\alpha$ energies between 1 and $2.5 \mathrm{MeV}$, since no error analysis was reported.

As performed in the present nuclear data library used in the SOURCES4C, and based on Bair's recommendation, the lower-energy ${ }^{17,18} \mathrm{O}(\alpha, \mathrm{n})$ cross sections were scaled by a large normalization factor of 1.35 applied to all data sets. This normalization of cross sections was performed on the basis of Bair's thick-target measurements of ${ }^{\text {nat }} \mathrm{O}(\alpha, \mathrm{n})[6]$ that had much smaller uncertainty. After these data sets were normalized, a new error of about $7 \%$ was assigned [6].

In Figs. 1 and 2, we show the ${ }^{17,18} \mathrm{O}(\alpha, \mathrm{n})$ cross section reconstructed from the preliminary set of resonance parameters in good agreement with the experimental data sets of Table I.

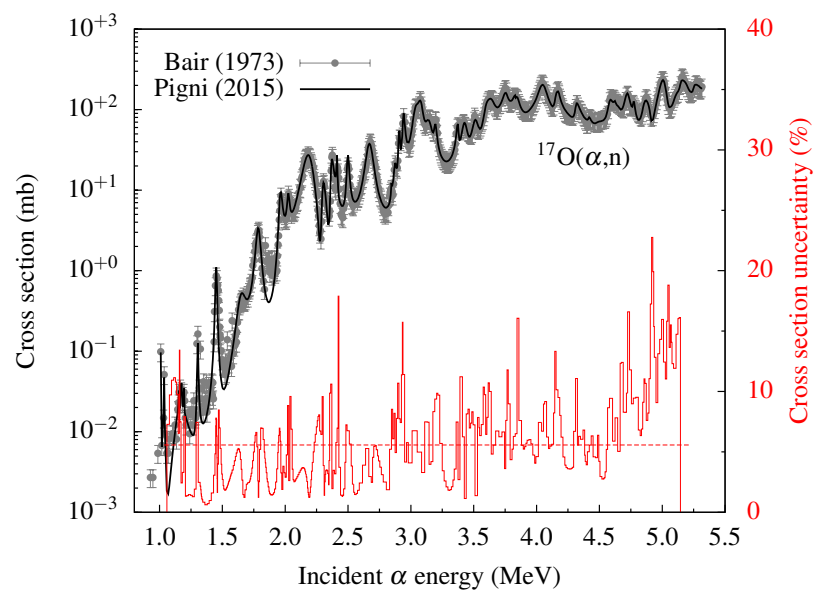

FIG. $1 .{ }^{17} \mathrm{O}(\alpha, \mathrm{n})$ cross sections reconstructed from the preliminary set of resonance parameters in the energy range of 1-5 MeV compared with Bair's experimental data [5]. Relative cross section uncertainties (in percent) are shown in a continuous red line, along with their average relative uncertainty $(5.6 \%)$ in a dashed red line.

In the analyzed energy range, as the result of the Bayesian update procedure used to update the preliminary set of parameters by taking into account the experimental data sets, we show the relative cross section

\footnotetext{
${ }^{1}$ The $(\alpha, \mathrm{n})$ reaction threshold for the most abundance isotope ${ }^{16} \mathrm{O}$ is not reached. Its energy threshold is $E_{\mathrm{th}}=15.1 \mathrm{MeV}$.
} 


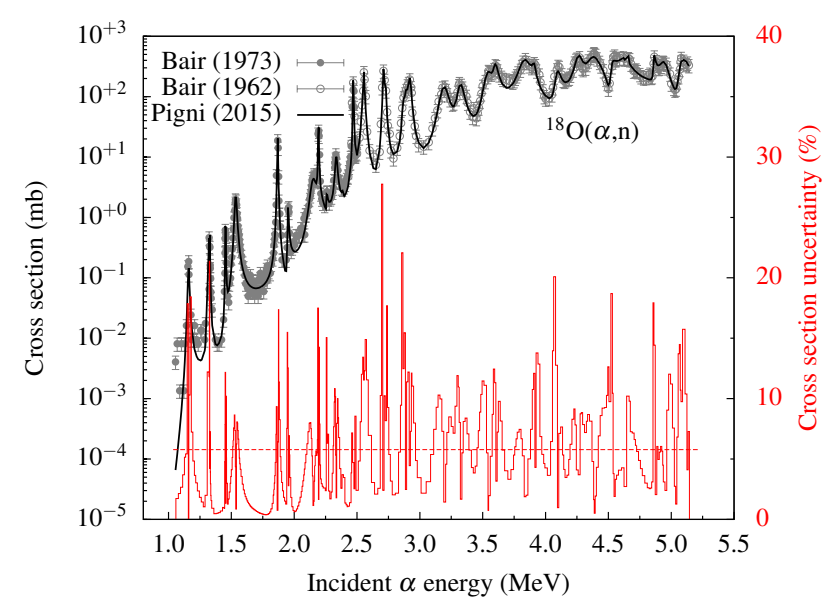

FIG. 2. ${ }^{18} \mathrm{O}(\alpha, \mathrm{n})$ cross sections reconstructed from the preliminary set of resonance parameters in the energy range of 1-5 MeV compared with Bair's experimental data [4] [5]. Relative cross section uncertainties (in percent) are shown in a continuous red line, along with their average relative uncertainty $(5.8 \%)$ in a dashed red line.

uncertainties (in a red continuous line). On average 5.6\% and $5.8 \%$ for ${ }^{17} \mathrm{O}$ and ${ }^{18} \mathrm{O}$, respectively, these uncertainties are smaller than those given in the experimental data as a consequence of the constraint from the energydependent correlations deriving from the $R$-matrix formalism [16] [17]. The physical constraint of the $R$-matrix formalism derives from the fact the collision matrix is unitary. This appears as the sharp and strong drop in the sensitivity or relative uncertainty near the each resonance peak (e.g., $E_{\alpha} \approx 1.8 \mathrm{MeV}$ for ${ }^{17} \mathrm{O}$ in Fig. 1). In other words, the reaction cross section is nearly independent of the model parameters very near the peak.

This feature is also seen in the cross section covariance matrices of Figs. 3 and 4 where the correlation from a very large positive value suddenly drops to a very large negative correlation. Additional constraints can be obtained by the simultaneous analysis of other reaction channels, such as elastic scattering; unfortunately, the elastic channels for these two oxygen isotopes are not available.

\section{B. Thick-target experimental data}

Bair's thick-target measurement of ${ }^{n a t} \mathrm{O}$ has an experimental uncertainty of $7 \%$ in the incident $\alpha$ energy range of 3.8-10.0 MeV (see Table I). As recommended in Ref. [6], this accurate measurement was used to normalize the thin-target ${ }^{17,18} \mathrm{O}(\alpha, n)$ cross-section data. As in the SOURCES4C data libraries, the same data for ${ }^{\text {nat }} \mathrm{O}(\alpha, \mathrm{n})$ were also used to adjust the high-energy ${ }^{17,18} \mathrm{O}(\alpha, \mathrm{n})$ cross section measurements of Hansen [7] by a $9.2 \%$ reduction, resulting from the integral data for ${ }^{\text {nat }} \mathrm{O}(\alpha, \mathrm{n})$ over the range $5.15 \leq E_{\alpha} \leq 8 \mathrm{MeV}[6]$.

In the present work, the ${ }^{18} \mathrm{O}(\alpha, \mathrm{n})$ cross sections above

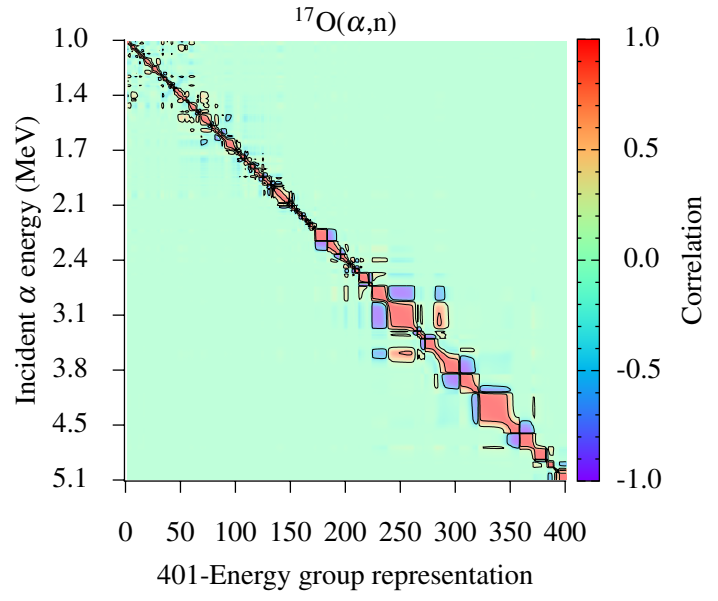

FIG. 3. Correlation matrix of ${ }^{17} \mathrm{O}(\alpha, n)$ cross sections in 401energy group representation. Correlations below $1 \%$ are not displayed.

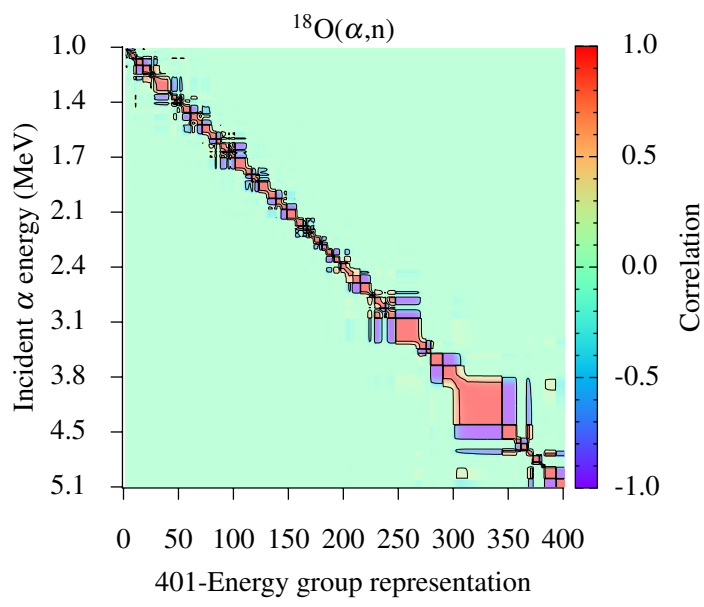

FIG. 4. Correlation matrix of ${ }^{18} \mathrm{O}(\alpha, n)$ cross sections in 401energy group representation. Correlations below $1 \%$ are not displayed.

$5.14 \mathrm{MeV}$ were adjusted to agree with West's experimental data $(\geq 3.8 \mathrm{MeV})$ for natural oxygen [11], which are of higher accuracy than Bair's measurements. In particular, we computed the average cross section from angleintegrated thick-target yield data $Y$ over the energy bin of $\Delta E=0.1 \mathrm{MeV}$ as

$$
\sigma(E)=\eta \cdot \frac{\bar{L}(E)}{w A} \cdot \frac{\partial Y(E)}{\partial E}
$$

where $\bar{L}$ is the average value of the mass stopping power $\left(\mathrm{MeV} \cdot \mathrm{cm}^{2} / \mathrm{g}\right)$ of an $\alpha$-particle in the $\mathrm{UO}_{2}$ sample with weight fraction $w$ and molar mass $\mathrm{A}$ of oxygen, and $\eta=$ 
$10^{27} / N_{\mathrm{A}}(\mathrm{mb} \cdot \mathrm{mol})$ is a conversion factor depending on the Avogadro number.

In Fig. 5, one can see that, as the energy increases, the resonance-like structure derived from Bair's experiments is replaced by a smooth, averaged behavior, yielding a cross section with broad fluctuations. Extracted from the thick-target yield data as defined in Eq. (6), the cross sections (red dots in Fig. 5) reveal a smooth energy dependence with distinct maxima and minima that are not seen in any other nuclear data cross section library (e.g., JENDL [10]). In the energy range between 1 and $5 \mathrm{MeV}$, Fig. 5 also displays the ${ }^{\text {nat }} \mathrm{O}(\alpha, n)$ cross section reconstructed with the SAMMY code from the set of parameters of ${ }^{17,18} \mathrm{O}$ isotopes, using their respective isotopic abundances. We also averaged these cross sections (black

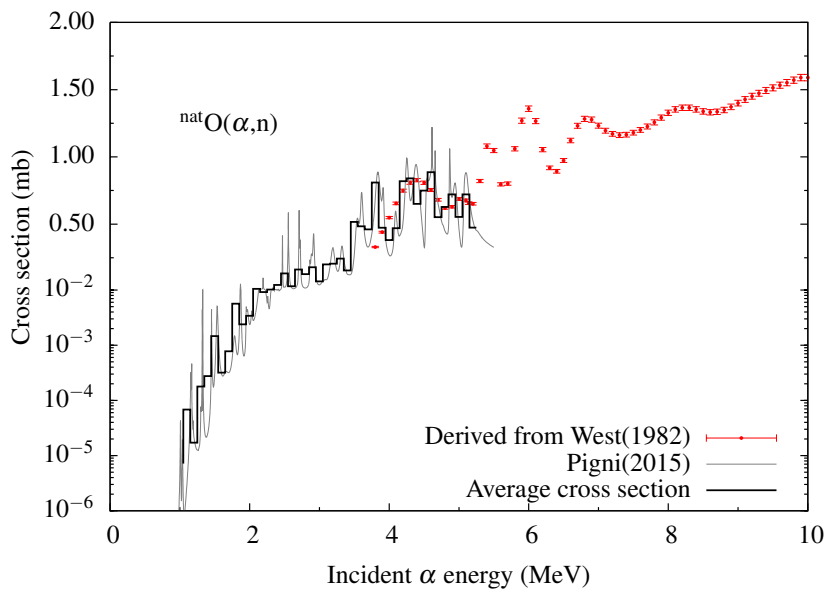

FIG. 5. ${ }^{\text {nat }} \mathrm{O}(\alpha, \mathrm{n})$ cross sections reconstructed from the preliminary set of ${ }^{17,18} \mathrm{O}(\alpha, \mathrm{n})$ resonance parameters in the energy range of $1-5 \mathrm{MeV}$ compared with Croft's cross sections derived from West's experimental data [11] (red dots). The black line also shows the reconstructed cross sections averaged over the same energy group structure used to obtain West's data.

line) over the energy bin $\Delta E=0.1 \mathrm{MeV}$ in order to ensure, for comparison with lower-resolution energy data, West's data to normalize the cross sections below $5 \mathrm{MeV}$. Integrating over the energy range 3.8 to $5 \mathrm{MeV}$, i.e., the energy range common to both data sets, we found the average cross sections (black line) were $3 \%$ lower than West's cross sections (red dots). The bias is consistent in view of the $\approx 6 \%$ uncertainty obtained by the Bayesian fitting with the stated $7 \%$ uncertainty of the low-energy Bair experimental data for the ${ }^{17,18} \mathrm{O}(\alpha, n)$ cross section. The black continuous line shows the average cross sections increased by the normalization factor of $3 \%$.

Moreover, since the isotopic abundance of ${ }^{18} \mathrm{O}$ makes the contribution of the ${ }^{17} \mathrm{O}(\alpha, n)$ cross section only about $10 \%$ to natural oxygen cross section, we estimated the ${ }^{18} \mathrm{O}$ cross sections above $5.14 \mathrm{MeV}$ as

$$
\beta_{18} \sigma\left({ }^{18} \mathrm{O}\right)=\sigma\left({ }^{\text {nat }} \mathrm{O}\right)-\beta_{17} \sigma\left({ }^{17} \mathrm{O}\right),
$$

where $\beta_{18}=0.00205$ and $\beta_{17}=0.00038$ are isotopic abundances for ${ }^{18} \mathrm{O}$ and ${ }^{17} \mathrm{O}$, respectively, and $\sigma\left({ }^{17} \mathrm{O}\right)$ is the ${ }^{17} \mathrm{O}(\mathrm{n}, \alpha)$ cross section adopted from JENDL evaluation.

\section{Stopping power cross sections}

The stopping power cross sections for each material and the $(\alpha, \mathrm{n})$ reaction cross sections for each isotope were used in SOURCES4C to compute the average number of emitted $(\alpha, \mathrm{n})$ neutrons as described in Section II through Eqs. (1)-(5). To quantify the contribution of the stopping power cross sections to the uncertainty of the output $(\alpha, n)$ source responses, stopping power cross sections based on ASTAR data [12] for oxygen and uranium were fitted to the analytic function

$$
\epsilon(E)=s_{\mathrm{L}}(E) \cdot s_{\mathrm{H}}(E) \cdot\left[s_{\mathrm{L}}(E)+s_{\mathrm{H}}(E)\right]^{-1},
$$

where, as used in the SOURCES4C code, the $s_{\mathrm{H}}$ and $s_{\mathrm{L}}$ (stopping power) functions are defined by a set of five coefficient factors, $c_{1}, \ldots, c_{5}$, as

$$
s_{\mathrm{H}}(E)=c_{1}(1000 \cdot E)^{c_{2}}
$$

and

$$
s_{\mathrm{L}}(E)=c_{3} E^{-1} \ln \left(1+c_{4} E^{-1}+c_{5} E\right),
$$

with the $\alpha$-energy $E$ in $\mathrm{MeV}$. In the fitting procedure the uncertainty of the ASTAR data was estimated as $2.5 \%$. The parameterization of ASTAR stopping power cross sections was also necessary because these data parameters are used by SOURCES4C to compute the reaction cross sections of natural oxygen in Eq. (6), from which we derived the normalization factor for the low-energy ${ }^{18} \mathrm{O}(\alpha, \mathrm{n})$ reaction cross sections. The results of the fitting procedure for oxygen and uranium are shown in Fig. 6, and the values of the related coefficients and uncertainty information are reported in Table II. These results were obtained using an implementation of the nonlinear leastsquares Marquardt-Levenberg algorithm.

\section{RESULTS}

The results shown in Fig. 7 were generated by performing 50 ORIGEN calculations each time randomly sampling cross section libraries. The set of 50 libraries was generated by random perturbation factors obtained from the multivariate (normal) distribution based on the covariance information relative to the evaluated ${ }^{17,18} \mathrm{O}(\alpha, \mathrm{n})$ cross sections in the $\alpha$-energy range between 1 and 10 $\mathrm{MeV}$. The same procedure was used to generate the perturbation factors for the coefficient factors reported in Table II used by ORIGEN to compute the stopping power cross sections for oxygen and uranium. We remark that, in the present work we used ORIGEN as a computational 


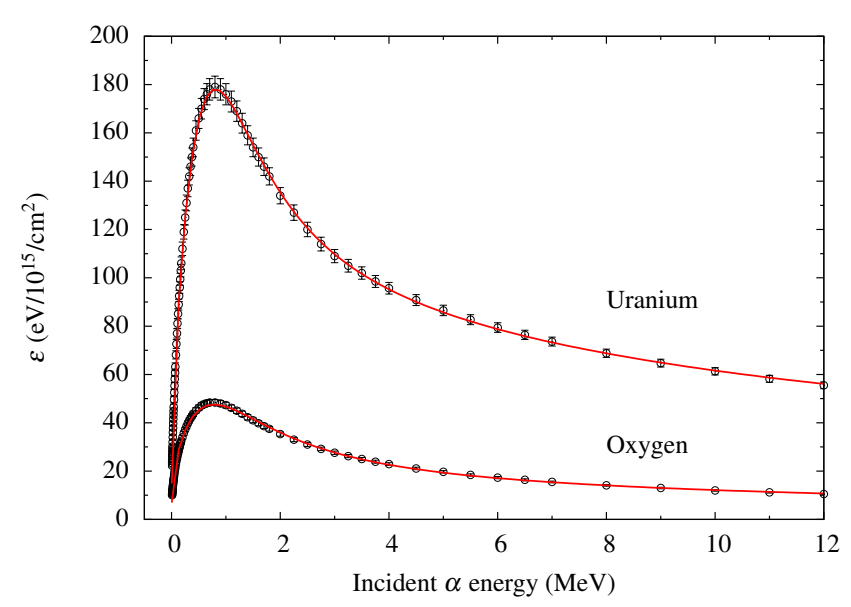

FIG. 6. Stopping power cross sections for oxygen and uranium obtained from the parameterization defined by Eq. (8) (in red) and ASTAR data (black dots).

TABLE II. Coefficient factors and related covariance information to compute from analytic functions of Eq. (8) the oxygen and uranium stopping power cross section of Fig. 6.

\begin{tabular}{|c|c|c|c|c|}
\hline \multicolumn{5}{|c|}{ oxygen } \\
\hline No. & $c_{i}$ & $\pm \Delta c_{i}(\%)$ & & Correlation matrix \\
\hline 1 & 3.439 & 1.12 & 1.000 & \\
\hline 2 & 0.438 & 0.62 & -0.967 & 1.000 \\
\hline 3 & 45.426 & 9.18 & 0.450 & -0.5461 .000 \\
\hline 4 & 17.111 & 23.21 & -0.364 & $0.449-0.9841 .000$ \\
\hline 5 & 1.458 & 26.84 & -0.459 & $\begin{array}{llll}0.556 & -0.996 & 0.971 & 1.000\end{array}$ \\
\hline \multicolumn{5}{|c|}{ uranium } \\
\hline No. & $c_{i}$ & $\pm \Delta c_{i}(\%)$ & & Correlation matrix \\
\hline 1 & 6.072 & 0.42 & 1.000 & \\
\hline 2 & 0.554 & 0.18 & -0.965 & 1.000 \\
\hline 3 & 368.5 & 2.55 & 0.302 & -0.3761 .000 \\
\hline 4 & 2.090 & 3.57 & -0.106 & $\begin{array}{lll}0.148 & -0.930 & 1.000\end{array}$ \\
\hline 5 & 0.472 & 5.06 & -0.306 & $\begin{array}{llll}0.381 & -0.995 & 0.908 & 1.000\end{array}$ \\
\hline
\end{tabular}

tool; but the SOURCES4C code has the same methods and capabilities in terms of $(\alpha, \mathrm{n})$ source calculations.

The precompiled data libraries of the SOURCES4C code are provided neither with cross section covariance information nor with stopping power covariances. Therefore, no uncertainty can be estimated for neutron source intensities. Based on the evaluation methodology described in Sec. III, we applied our approach to estimate the uncertainty in the neutron generation for a typical $\mathrm{UO}_{2}$ fuel type for which the $\alpha$-particles emitted by uranium isotopes interact with ${ }^{17,18} \mathrm{O}$ to produce neutrons. The error bar with continuous red line in Fig. 7 shows that the covariance information applied only to ${ }^{17,18} \mathrm{O}$ reaction cross sections (for an average uncertainty of about $6 \%$ ) leads to about $0.5 \%$ uncertainty in the total $(\alpha, \mathrm{n})$ source. We also show in the error bar with the dashed red lines the case for which the stopping power cross section uncertainty of oxygen and uranium was included by the sampling of the coefficient factors and related covariance information in the ORIGEN calculations as well. In this scenario, the total uncertainty is almost doubled (about $\pm 1 \%$ ) compared with the uncertainty from the cross sections alone. The deviation of the West data [11] compared with the ORIGEN calculations are shown (blue square and red dots in Fig. 7).

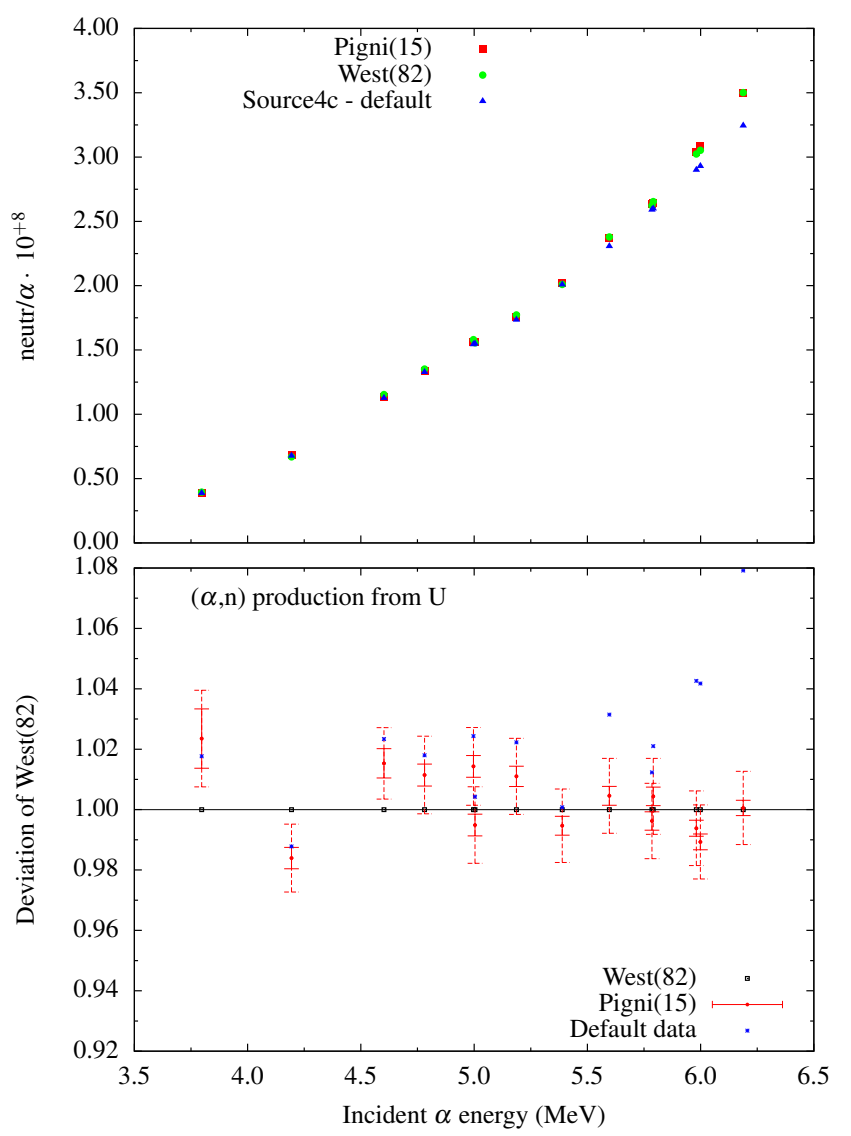

FIG. 7. (Top) Results of $(\alpha, n)$ neutron production for default calculations (in blue) comparing the West data (in green) and the present calculations (in red). (Bottom) Uncertainties for $(\alpha, \mathrm{n})$ neutron production from uranium computed from covariance information for the reaction cross section of ${ }^{17,18} \mathrm{O}(\alpha, \mathrm{n})$ and stopping power cross section for oxygen and uranium. The deviation of the West data [11] from the default (in blue) and present (in red) calculations is also shown.

\section{CONCLUSIONS}

We have developed a self-consistent and rigorous methodology to propagate nuclear covariance data to neutron source calculations using the ORIGEN code. Particularly, our work represents the first attempt to gen- 
erate covariance information for both $(\alpha, n)$ cross sections and stopping power cross sections.

First, we produced estimates of $(\alpha, \mathrm{n})$ cross section covariances for ${ }^{17,18} \mathrm{O}$ in the energy range between 1 and $10 \mathrm{MeV}$. Up to about $5 \mathrm{MeV}$, the covariance information is based on the Bayesian fitting method implemented in the $R$-matrix SAMMY code and on selected experimental data sets. Above about $5 \mathrm{MeV}$, our results are based on experimental cross sections for natural oxygen for ${ }^{18} \mathrm{O}$, as well as evaluated cross sections taken from the JENDL nuclear data library for ${ }^{17} \mathrm{O}$. Based on the analysis of natural oxygen data, we applied a $+3 \%$ normalization factor to the ${ }^{17,18} \mathrm{O}$ cross section below about $5 \mathrm{MeV}$. We also estimated covariance information for the stopping cross sections for oxygen and uranium. This task was performed by the fit of stopping power cross sections based on ASTAR data [12], i.e., the same data used to compute the reaction cross section for natural oxygen, so as to avoid introducing an inconsistency bias.

Second, a set of 50 data libraries sampled from the generated covariances was used in ORIGEN calculations to obtain a set of randomly perturbed output responses, such as the $(\alpha, \mathrm{n})$ neutron source. Finally, in the statistical analysis of the output results that provided the mean values and standard deviations in the calculated responses, we found that covariance information applied to ${ }^{17,18} \mathrm{O}$ reaction cross sections leads only to about $0.5 \%$ uncertainty in the total $(\alpha, \mathrm{n})$ neutron source, in this case under the assumptions described. When the covariance information was also applied to the stopping power cross sections for oxygen and uranium, the total uncertainty increased to about 1\%. As shown in Fig. 7, the uncertainty related to our calculations is in most cases within the deviation of the data.

The method is readily extendable to other matrices such as $\mathrm{PuO}_{2}$, although this would also require extrapolation of stopping power data to Plutonium. The method is also applicable to other reactions. To obtain the full benefit, experimental covariance information is needed, for instance, if the neutron efficiency is not flat; however, to date insufficient information is available to realistically include this. Future thin-target measurements should attempt to report covariance information.

\section{ACKNOWLEDGMENTS}

The authors express their gratitude to Dr. Luiz C. Leal for triggering the methodology described in the paper and stimulating discussions in this project.

This work was supported by the U.S. Department of Energy, National Nuclear Security Administration Office of Defense Nuclear Nonproliferation Research and Development.
[1] I. C. Gauld et al., "Isotopic Depletion and Decay Methods and Analysis Capabilities," Nucl. Tech. 174, 169 (2011).

[2] W. B. Wilson et al., Report LA-UR-02-1839, Los Alamos National Laboratory, Los Alamos, NM (2002). R. T. Perry et al., Report LA-8869-MS, Los Alamos National Laboratory, Los Alamos, NM (1981).

[3] I. C. Gauld et al., Proc. of the ANS $12^{\text {th }}$ Biennial RPSD Topical Meeting, Santa Fe, New Mexico, April 14, (2002).

[4] J. K. Bair et al., Level Structure in ${ }^{22} \mathrm{Ne}$ and ${ }^{30}$ Si from the Reactions ${ }^{18} \mathrm{O}(\alpha, n)^{21} \mathrm{Ne}$ and ${ }^{26} \mathrm{Mg}(\alpha, n)^{29} \mathrm{Si}$, Phys. Rev. 128, 299 (1962).

[5] J. K. Bair et al., "Total Neutron Yield from the Reactions ${ }^{13} \mathrm{C}(\alpha, n)^{17} \mathrm{O}$ and ${ }^{17} \mathrm{O},{ }^{18} \mathrm{O}(\alpha, n)^{20} \mathrm{Ne},{ }^{21} \mathrm{Ne}, "$ Phys. Rev. C7, 1356 (1973).

[6] J. K. Bair et al., "Neutron Yields from Alpha-Particle Bombardment," Nucl. Sci. Eng. 71, 18 (1979).

[7] L. S. Hansen et al., "The $(\alpha, n)$ cross sections on ${ }^{17} O$ and ${ }^{18} \mathrm{O}$ between 5 and 12.5 MeV," Nucl. Phys. A98, 25 (1967).

[8] R. Babut et al., "Reich-Moore Parameterization of $(\alpha, n)$
Reactions on Light Nuclei: Impact on a Neutron Source Calculation in an Oxide Fuel," Nucl. Sci. Eng. 151, 135 (2005).

[9] N. M. Larson, Report ORNL/TM-9179/R8, Oak Ridge National Laboratory, Oak Ridge, TN (October 2008).

[10] T. Murata et al., JAEA-Research 2006-052 (July 2006).

[11] D. West et al., "Measurements of Thick-Target $(\alpha, n)$ Yields from Light Elements," Ann. Nucl. Ene. 9, 551 (1982).

[12] National Institute of Standards and Technology (NIST) Physical Meas. Laboratory ASTAR database.

[13] W. H. Bragg et al., "On the Ionization Curves of Radium," Philosophical Magazine 8, 726 (1904).

[14] F. H. Fröhner, JEFF Report 18 (2000).

[15] M. T. Pigni et al., ICNC2015 proceedings (accepted for publication).

[16] S. Kunieda et al., "Covariance of Neutron Cross Sections for ${ }^{16} O$ through R-matrix Analysis," Nuclear Data Sheets 123, 159 (2015).

[17] M. Herman et al., Report BNL-80173-2008 (April 2008). 